\title{
Utilisation des fantômes numériques voxélisés pour l'amélioration des étalonnages en anthroporadiamétrie pulmonaire
}

\author{
N. PIERRAT ${ }^{1}$, L. DE CARLAN ${ }^{1}$, D. FRANCK ${ }^{1}$
}

(Manuscrit reçu le 20 février 2005, accepté le 19 avril 2005)

RÉSUMÉ L'anthroporadiamétrie pulmonaire est une méthode de choix pour la surveillance des travailleurs de l'industrie nucléaire exposés à un risque de contamination interne. Un certain nombre de difficultés est cependant rencontré avec cette technique, difficultés principalement liées aux conditions d'étalonnage, conduisant à des incertitudes et des erreurs systématiques importantes sur les résultats. En effet, l'utilisation de fantômes physiques d'étalonnage reste un facteur limitant et engendre d'importantes corrections en vue d'une extrapolation à un individu donné. Une perspective prometteuse pour diminuer les erreurs systématiques consiste à étalonner de façon individuelle le système de mesure avec des fantômes numériques spécifiques aux personnes à mesurer. Dans ce but, une interface appelée CEDIPE (Outil d'Évaluation de la Dose Interne PErsonnalisée), associant fantômes numériques voxélisés et calcul Monte Carlo (MCNP), a été développée au Laboratoire d'Évaluation de la Dose Interne de l'IRSN. Le but de l'étude proposée ici est de montrer le potentiel de cette technique pour la simulation réaliste de la mesure anthroporadiamétrique des actinides dans les poumons. Après la présentation de l'interface CEDIPE pour la mesure pulmonaire, sa validation est présentée en utilisant un fantôme d'étalonnage couramment utilisé (fantôme Livermore). Ensuite, une comparaison de différents fantômes thoraciques d'étalonnage par rapport au fantôme numérique d'une personne (fantôme de Zubal) a été effectuée afin de montrer les variations d'étalonnage engendrées par les différences morphologiques de ces fantômes et donc la nécessité d'un étalonnage plus spécifique par individu. Enfin, des approches de contaminations inhomogènes ont été réalisées afin de montrer l'intérêt de la technique pour l'étude des variations des facteurs d'étalonnage en fonction de la répartition des radioéléments dans les poumons. Les différents résultats montrent le potentiel de cette technique pour l'étalonnage réaliste des installations anthroporadiamétriques.

ABSTRACT Use of voxel phantoms for the improvement of lung counting calibrations.

In vivo lung counting is one of the preferred methods for the monitoring of nuclear workers exposed to a risk of internal contamination. Some difficulties are still encountered while using this technique, mainly due to calibration conditions, leading to large uncertainties and important systematic errors on results. Indeed, the use of physical calibration phantoms remains a limiting factor and generates large corrections for the extrapolation to a given subject. A promising perspective for decreasing systematic errors is based on a subject-specific calibration method using

\footnotetext{
${ }^{1}$ IRSN, DRPH/SDI/LEDI, B.P. 17, 92262 Fontenay-aux-Roses Cedex, France
} 


\begin{abstract}
numerical phantoms. In this context, an interface called CEDIPE (French acronym for "tool for internal personalized dose assessment"), associating numerical voxel phantoms and Monte Carlo calculation (MCNP), was developed in the laboratory. The purpose of this work is to show the potential of this technique for the realistic simulation of in vivo lung measurement of actinides. After the presentation of the CEDIPE software for lung measurement, its validation will be presented using a calibration phantom currently used (Livermore phantom). Then a comparison between different thoracic calibration phantoms and a numerical phantom of a person (Zubal phantom) was made in order to show calibration variations generated by morphological differences of these phantoms and thus to prove the necessity of a more specific calibration for a given subject. Finally, approaches of heterogeneous contaminations were realized to show the interest of the technique for the study of the variations of calibration factors according to the distribution of radionuclides in lungs. All results show the power of the technique for realistic calibration of in vivo systems.
\end{abstract}

Keywords: lung counting / Monte Carlo / numerical phantom / uncertainties / heterogeneous contamination

\title{
1. Introduction
}

La mesure anthroporadiamétrique consiste à détecter et à quantifier le niveau d'activité d'un ou plusieurs radionucléides contenus dans le corps entier ou dans un organe particulier en effectuant une mesure spectrométrique externe d'une personne et en la comparant à celle réalisée à l'aide d'un fantôme d'étalonnage. Une contamination interne par actinides s'avère particulièrement complexe à détecter ; en effet, ces éléments sont des émetteurs $\alpha$, ne pouvant être détectés par mesure externe que via les désintégrations $\mathrm{X}$ et $\gamma$ qui leur sont associées. Or, ces photons émis sont de faible énergie $(<200 \mathrm{keV})$ et sont donc très fortement absorbés dans les tissus thoraciques ; les probabilités d'émission de ces photons sont très faibles, ce qui a pour conséquence une faible sensibilité de détection (en particulier pour le ${ }^{239} \mathrm{Pu}$ ). De plus, les fantômes physiques utilisés lors de l'étalonnage ne peuvent représenter pour des raisons techniques qu'une géométrie approximative de la personne à mesurer et restent limités par l'hypothèse que la distribution de la contamination est toujours homogène dans les poumons.

De ce fait, un des problèmes majeurs de la mesure pulmonaire réside dans les incertitudes associées à la mesure des rayonnements (en particulier les photons d'énergie inférieure à $20 \mathrm{keV}$ ), liées à l'atténuation, ainsi que dans les erreurs systématiques sur les résultats engendrées par l'utilisation de fantômes physiques (Franck et al., 1997). L'étalonnage est donc un facteur déterminant lors d'une estimation de contamination pulmonaire par actinides. De plus, la connaissance de l'épaisseur et de la composition chimique des tissus de la paroi thoracique est cruciale. En général, cette épaisseur est estimée en utilisant des techniques d'ultrasons ou des mesures bioparamétriques utilisant essentiellement le poids et la taille (Vickers, 1996) qui sont d'une précision insuffisante pour permettre une 
correction satisfaisante des facteurs d'étalonnage. C'est pourquoi il est important de développer de nouvelles techniques d'étalonnage prenant plus facilement en compte ce type de variations. De précédents travaux ont déjà montré la possibilité de tels étalonnages par les techniques de Monte Carlo (Mallett et al., 1995 ; Hunt et al., 1998) ; cependant, ces équipes utilisent des fantômes numériques prédéfinis et sont donc limitées par le nombre restreint de ces derniers.

Ce travail, présenté en trois grandes parties, se propose d'aller plus loin par la combinaison de fantômes voxélisés ${ }^{2}$ spécifiques aux personnes avec le code Monte Carlo MCNP (Briesmeister, 2000). Afin de réaliser de façon simple et automatique la simulation, une interface graphique (appelée « (EDIPE») a été spécialement développée (Franck et al., 2001 ; Borisov et al., 2002 ; de Carlan et al., 2003). Après une rapide présentation de cet outil, la validation de la méthode sera exposée pour une configuration réaliste multidétecteurs à l'aide du fantôme anthropomorphe de Livermore. Ensuite, la comparaison entre différents fantômes d'étalonnage utilisés dans le monde et un fantôme numérique fondé sur les images d'une personne réelle (fantôme de Zubal) sera présentée. Enfin un modèle simple d'approche d'une contamination inhomogène dans les poumons sera proposé.

\section{Présentation d'CEDIPE}

Une interface graphique appelée CEDIPE (Outil d'Évaluation de la Dose Interne Personnalisée) est développée au laboratoire en langage PV-WAVE® (Franck et al., 2001 ; Borisov et al., 2002). Cet outil convivial permet soit de réaliser une simulation de mesure anthroporadiamétrique (Franck et al., 2003), soit d'effectuer un calcul de dose (fonctionnalité non détaillée dans cet article) (Aubineau-Lanièce et al., 2004). Le principal intérêt de cet outil est de créer de façon automatique le fichier d'entrée MCNP à partir de différents paramètres nécessaires à la simulation. La figure 1 décrit les fonctionnalités d'EDDIPE.

- La première étape de la simulation consiste à définir un fantôme numérique. Pour cela, des images CT scanner ou IRM de la personne à mesurer ou du fantôme à étudier sont réalisées. Ces images sont ensuite importées dans l'interface CEDIPE ( $c f$. Fig. 1-(1) où la segmentation ${ }^{3}$ est alors réalisée en deux étapes. La première étape consiste à délimiter de façon géométrique sur les images anatomiques les organes ou tissus d'intérêt; cette fonctionnalité est possible grâce à un module de traitement des images qui permet de séparer les différents niveaux de gris qui les composent et donc de définir des « zones » de

\footnotetext{
${ }^{2}$ Ces fantômes sont reconstruits à partir d'images CT scanner (Computed Tomography) ou IRM constituées de pixels qui, une fois intégrés en 3 dimensions, deviennent de petits éléments de volumes ou voxels, d'où le nom de fantômes numériques voxélisés.

${ }^{3}$ La segmentation consiste à différencier des zones de couleur sur des images anatomiques, en fonction du niveau de gris par exemple, et à associer à chaque voxel de ces zones un organe ou un tissu donné.
} 
couleurs. Dans un second temps, sont affectées à ces «zones » les propriétés physiques et chimiques des organes ou tissus délimités (composition chimique, densité), décrites par la Commission internationale pour les unités de rayonnements et les mesures (ICRU, 1989).

- Une source est ensuite définie, de type homogène dans un tissu ou ponctuelle (la localisation de la contamination est alors précisée en cliquant sur le voxel souhaité), en spécifiant les radionucléides utilisés, les activités associées et le temps de comptage. L'étape suivante consiste à choisir un ou plusieurs détecteurs dans une bibliothèque (où sont stockées toutes les caractéristiques géométriques et intrinsèques des détecteurs proposés) et à les positionner par rapport au fantôme numérique afin de reproduire une mesure physique le plus fidèlement possible.

- À partir de ces données, EDIPE va générer de façon automatique le fichier d'entrée pour le code Monte Carlo MCNP ( $c f$. Fig. 1-2), cette fonctionnalité représentant son principal atout.

- L'exploitation du fichier de sortie issu du calcul Monte Carlo MCNP ( $c f$. Figs. 1-3 et 1-4) est également possible avec EDIPE. Les résultats sont alors visualisés sous forme de courbes d'isodébits de dose pour la partie «calcul de dose », ou sous la forme d'un spectre pour la partie «simulation de mesure anthroporadiamétrique » ( $c f$. Fig. 1-5). Le spectre calculé peut alors être superposé au spectre mesuré, ce qui permet une comparaison aisée entre mesure et simulation associée.

La segmentation étant une étape critique du processus de simulation, le logiciel DOSIGRAY $^{\circledR}$ a été installé en parallèle de l'interface CEDIPE afin d'optimiser cette étape. Ce logiciel, commercialisé par la société DosiSoft ${ }^{\circledR}$, est un système de planification de traitement utilisé en radiothérapie pour calculer les doses délivrées aux patients ; dans le cadre de nos études, seules ses potentialités pour le traitement de l'image ont été exploitées. En effet, cette interface permet d'effectuer de façon rapide des contours semi-automatiques des organes ou tissus d'intérêt directement à partir des images tomographiques (scanner ou IRM) ; il a ainsi été possible de définir les poumons, les os, le muscle, la graisse... Une passerelle a donc été créée entre DOSIGRAY ${ }^{\circledR}$ et l'interface CEDIPE pour pouvoir exploiter directement ces contours. D'utilisation simple et pratique, ce logiciel permet un gain de temps substantiel lors de l'étape de segmentation.

\section{Validation d'CEDIPE pour la mesure anthroporadiamétrique multidétecteurs}

L'interface CEDIPE a été dans un premier temps développée puis validée pour un système monodétecteur (Borisov et al., 2002). La configuration la plus souvent 

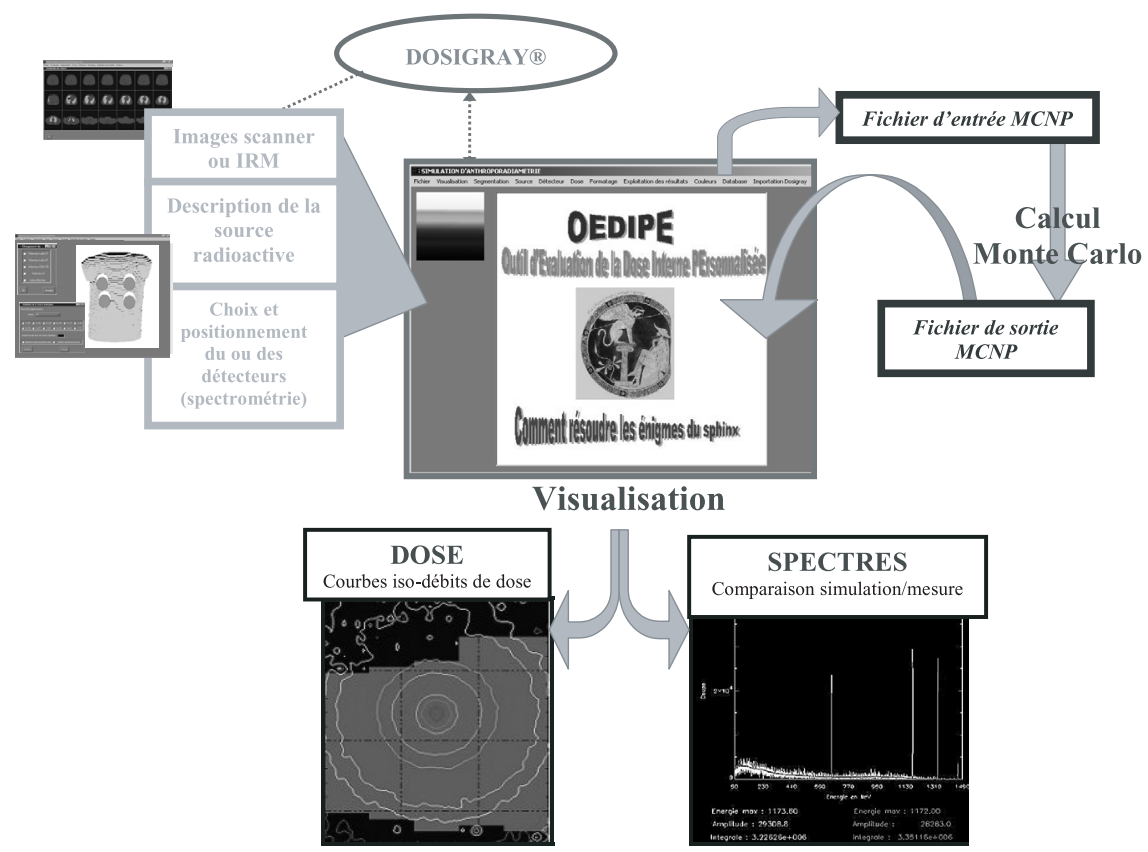

Figure 1 - Description du fonctionnement du logiciel EDIPE : 1 entrée des données nécessaires à la création du fichier d'entrée MCNP (fantôme, source(s), détecteur(s), géométrie de détection), 2 création automatique du fichier d'entrée MCNP, 3 calcul MCNP, (4) lecture et extraction des données pertinentes du fichier de sortie MCNP, 5 visualisation des données (dose absorbée ou spectre de dépôt d'énergie dans le détecteur).

Description of EDIPE software: 1 input data which are required for the creation of MCNP input file (phantom, source(s), detector(s), detection geometry), 2 automatic creation of MCNP input file, 3 MCNP calculation, 4 processing of the MCNP ouput file, 5 data visualization (absorbed dose or spectrum of deposited energy in the detector).

employée en routine étant 4 détecteurs, un module particulier a été développé dans CEDIPE afin d'accepter plusieurs détecteurs en entrée. Cette évolution a ensuite été validée en simulant une installation réelle comportant 4 détecteurs, comme cela est présenté ci-après.

\subsection{Matériel et méthodes}

\subsubsection{Fantôme}

Le fantôme d'étalonnage utilisé lors des mesures est le fantôme de Livermore qui a été spécialement conçu pour l'étalonnage des systèmes de mesure 
a)

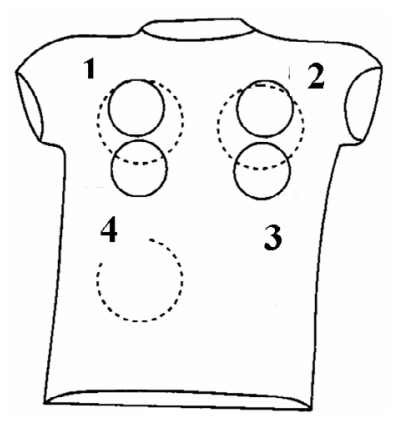

b)

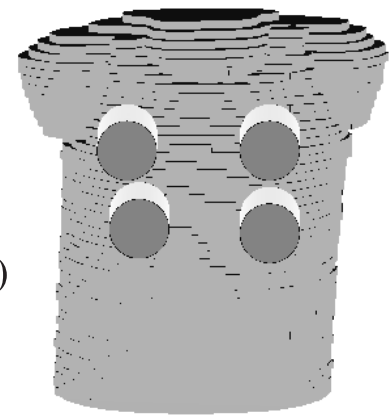

Figure 2 - Positionnement des détecteurs germanium par rapport au fantôme Livermore : (a) littérature (Kramer et al., 1998), (b) visualisation de la géométrie de simulation par Sabrina ${ }^{\circledR}$. Positioning of germanium detectors related to Livermore phantom: (a) literature, (b) visualization of simulation geometry by Sabrina ${ }^{\circledR}$.

anthroporadiamétrique basse énergie (Griffith et al., 1978 ; Newton et al., 1985). Ce fantôme présente des poumons amovibles uniformément contaminés par un radionucléide d'intérêt et des plaques extrathoraciques qui permettent de simuler différentes morphologies lors de l'étalonnage.

Lors des mesures, le fantôme était équipé de la plaque $\mathrm{n}^{\circ} 0$ (matériau équivalent $100 \%$ muscle ; épaisseur : $1,9 \mathrm{~cm}$ ) à laquelle s'ajoutait la plaque extrathoracique $\mathrm{n}^{\circ} 3$ (matériau équivalent $50 \%$ muscle $-50 \%$ graisse ; épaisseur : $1,6 \mathrm{~cm})$, ce qui représente une épaisseur thoracique physique totale de $3,5 \mathrm{~cm} . \mathrm{Ce}$ fantôme contenait tour à tour des poumons contaminés en ${ }^{241} \mathrm{Am}(70 \mathrm{kBq})$ et en ${ }^{235} \mathrm{U}$ enrichi à $93 \%$ (80 Bq).

\subsubsection{Système de détection et mesure}

Le système de mesure du laboratoire est composé d'un détecteur germanium de haute pureté (LEGe de Canberra), de 50,5 $\mathrm{mm}$ de diamètre et $20 \mathrm{~mm}$ d'épaisseur avec une fenêtre d'entrée de $0,5 \mathrm{~mm}$ en époxy et carbone. Afin de simuler une configuration multidétecteurs, ce détecteur a été positionné à 4 reprises au-dessus du fantôme d'étalonnage, selon une configuration typique choisie dans la littérature et présentée sur la figure 2a (Kramer et al., 1998).

Pour chaque mesure, un temps de comptage de 3600 secondes a été choisi.

\subsubsection{Simulation}

Après segmentation des 49 images scanner (épaisseur de coupe $=1 \mathrm{~cm}$ ) du fantôme Livermore équipé de la plaque $\mathrm{n}^{\circ} 3$, le fantôme numérique a été 
reconstruit par EDDIPE suivant la méthode décrite précédemment. La résolution des images est de $256 \times 256$ pixels sur 49 coupes ; la taille de chaque voxel est de $1,94 \times 1,94 \times 10 \mathrm{~mm}^{3}$.

Tous les paramètres simulés sont identiques à ceux utilisés lors de la mesure physique. Ainsi, les sources de $70 \mathrm{kBq}$ en ${ }^{241} \mathrm{Am}$ et de $80 \mathrm{~Bq}$ de ${ }^{235} \mathrm{U}$ simulées dans le fantôme numérique ont été réparties de façon homogène dans les poumons, le temps de comptage de 3600 secondes et la géométrie de détection similaire (visualisée par le logiciel Sabrina ${ }^{\circledR}$ sur la Fig. 2b).

\subsection{Résultats}

La comparaison par CEDIPE des spectres calculé et mesuré avec une source de

${ }^{241} \mathrm{Am}$ pour la position 1 (partie supérieure du poumon droit) est présentée à la figure 3. La comparaison de l'ensemble des résultats calculés avec les mesures physiques est donnée dans le tableau I (colonne «initial»), en considérant uniquement les pics photoélectriques à $59,54 \mathrm{keV}$ pour ${ }^{241} \mathrm{Am}$ et $185,72 \mathrm{keV}$ pour ${ }^{235} \mathrm{U}$.

De façon assez surprenante, à 185,72 keV, les calculs sous-estiment de $20 \%$ en moyenne la mesure alors que l'inverse était attendu à cause des pertes de collection de charges non modélisées. Les résultats en ${ }^{241} \mathrm{Am}$ semblent quant à eux plus cohérents, sauf pour le détecteur 3 (partie inférieure du poumon gauche) pour lequel un écart de plus de $25 \%$ est observé entre simulation et mesure, explicable par un possible mauvais positionnement dans CEDIPE engendrant ainsi une importante erreur sur le résultat de la simulation. Cette hypothèse semble vraisemblable au vu des résultats pour l'uranium qui montrent une sous-estimation de près de $30 \%$ pour ce même détecteur 3 .

\subsection{Optimisation}

Afin de mieux appréhender ce problème, les positions de chaque détecteur ont été légèrement modifiées : pour être encore plus représentatifs de la mesure physique réalisée, les détecteurs positionnés sur la partie basse des poumons ont été déplacés de $2 \mathrm{~cm}$ vers la tête et ceux situés sur la partie haute de $5 \mathrm{~mm}$ (avec un décalage latéral vers le sternum de $8 \mathrm{~mm}$ pour le détecteur 1). De tels écarts peuvent sembler importants mais sont typiquement du même ordre de grandeur que l'erreur commise entre la mesure et la simulation de cette même géométrie dans EDIPE, et également en mesure réelle. Les nouveaux résultats obtenus sont présentés dans le tableau I (colonne « après modification »).

Après cette modification de positionnement, la concordance entre simulation et mesure est meilleure pour la plupart des détecteurs, hormis le détecteur 1 qui 


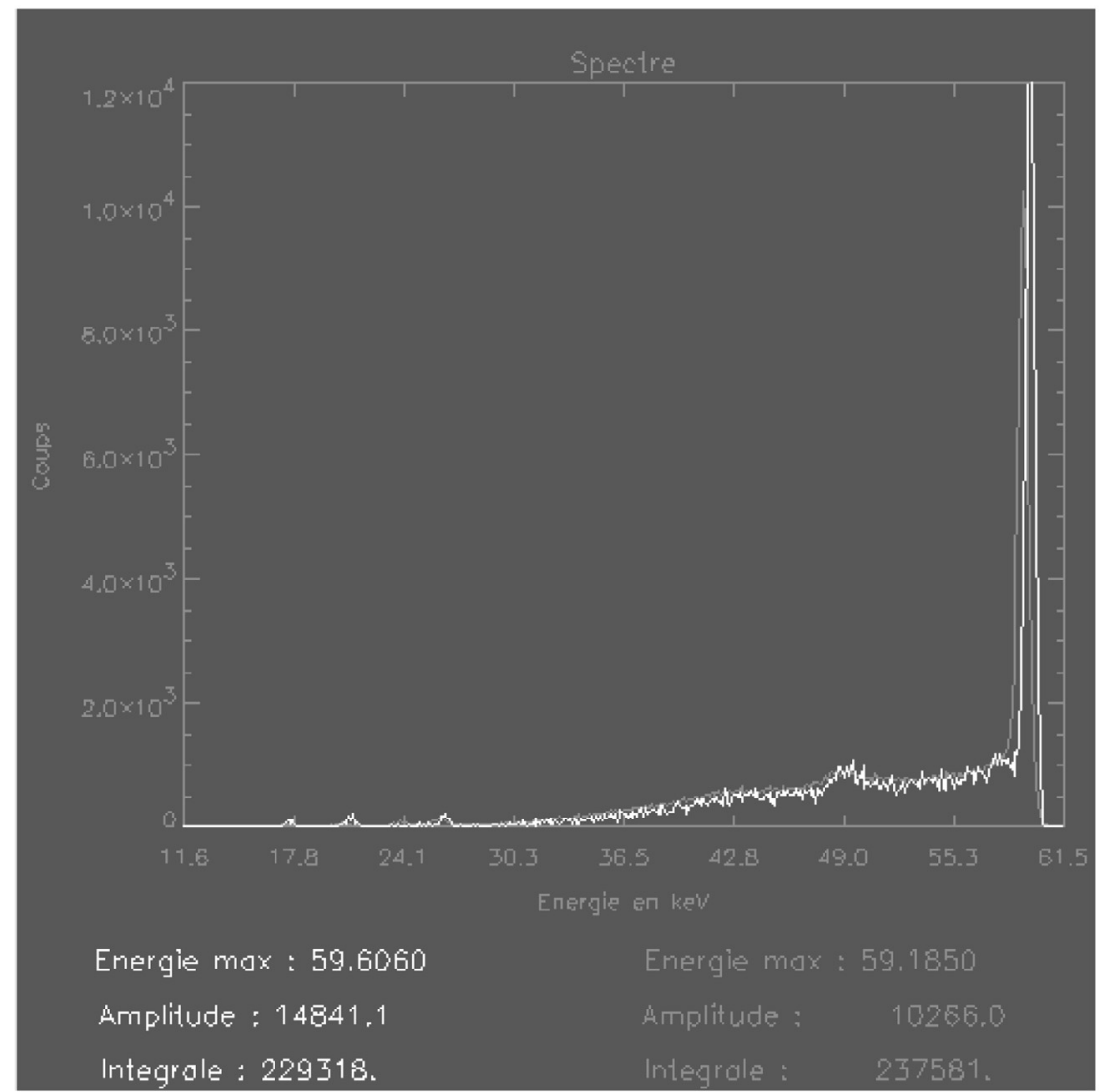

Figure 3 - Comparaison avec CEDIPE du spectre simulé (blanc) et du spectre expérimental (gris) pour la position 1 (poumon droit haut).

Comparison by CEDIPE of simulated spectrum (white) with measured spectrum (grey) for the position 1 (upper right lung).

montre une légère dégradation en ${ }^{241} \mathrm{Am}$ pour des modifications de positionnement assez faibles. Il est donc possible de conclure que la sensibilité de positionnement des détecteurs constitue un paramètre critique pour l'étalonnage de la mesure des émetteurs basse énergie.

Par ailleurs, les faibles écarts entre simulation et mesure en ${ }^{241} \mathrm{Am}$, notamment sur la somme des quatre détecteurs ( $2 \%$ d'écart entre simulation et mesure après modification de positionnement), montrent que la validation de la méthode n'est pas remise en question pour un système multidétecteurs. Cependant, d'importants 
TABLEAU I

Écarts observés entre simulation et mesure pour les pics à $59,54 \mathrm{keV} \mathrm{de}{ }^{241} \mathrm{Am}$ et à $185,72 \mathrm{keV}$ de ${ }^{235} \mathrm{U}$ pour une mesure 4 détecteurs à l'aide du fantôme Livemore, avant et après modification de positionnement des détecteurs.

Differences between simulation and measurement for the $59.54 \mathrm{keV}$ peak of ${ }^{241} \mathrm{Am}$ and the $185.72 \mathrm{keV}$ peak of ${ }^{235} \mathrm{U}$ for a 4 detectors measurement with Livermore phantom, before and after the modification of detectors positioning.

\begin{tabular}{lcccc}
\hline & \multicolumn{4}{c}{ Rapport simulation / mesure } \\
\cline { 2 - 5 } & \multicolumn{2}{c}{${ }^{241} \mathrm{Am}(59,54 \mathrm{keV})$} & ${ }^{235} \mathrm{U}(185,72 \mathrm{keV})$ \\
\hline Position du détecteur & Initial & Après modification & Initial & Après modification \\
1 & 1,04 & $\mathbf{1 , 1 3}$ & 0,77 & 0,75 \\
2 & 0,84 & 0,91 & 0,84 & 0,89 \\
3 & $\mathbf{0 , 7 4}$ & 1,04 & $\mathbf{0 , 7 2}$ & 0,86 \\
4 & 0,94 & 1,01 & 0,85 & 0,95 \\
Somme & 0,91 & 1,02 & 0,80 & 0,86 \\
\hline
\end{tabular}

écarts sont encore constatés avec la source en ${ }^{235} \mathrm{U}$ : la simulation sous-estime la mesure de $15 \%$ en moyenne, pouvant atteindre jusqu'à $25 \%$ pour le détecteur 1 , alors que l'uranium, d'énergie plus élevée que l'américium, devrait être moins critique pour les problèmes de positionnement. Ces résultats laisseraient donc supposer un problème au niveau des poumons eux-mêmes, hypothèse discutée dans le paragraphe suivant.

\section{4. Étude spécifique des poumons uranium}

Des difficultés ont déjà été rencontrées sur les poumons et sont sans doute liées à la méthode de fabrication de ces derniers, qui consiste en un mélange manuel des radionucléides dans la mousse (Spitz et al., 1994 ; Kramer et Hauck, 2000). Pour cette raison, une étude comparative de 3 paires de poumons uranium a été entreprise : une paire en ${ }^{235} \mathrm{U}$ enrichi à $3 \%$, une autre paire enrichie à $93 \%$ et enfin une paire de poumons dits « $\mathrm{MOX} »$. Cette dernière paire, fabriquée à la demande $\mathrm{du}$ laboratoire, contient un mélange d'actinides, en particulier ${ }^{235} \mathrm{U},{ }^{238} \mathrm{Pu}$ et ${ }^{241} \mathrm{Am}$.

Chaque jeu de poumons a été pesé puis compté dans une même géométrie pendant 16 heures. Leurs caractéristiques physiques et leurs efficacités (mesurée et calculée) sont présentées dans le tableau II. L'efficacité absolue du système est définie comme étant le rapport du nombre de coups mesurés sur le nombre théorique de photons émis.

D'importantes disparités peuvent être notées tant au point de vue de la densité qu'au niveau de l'efficacité absolue pour chacun des jeux de poumons. En ce qui 


\section{Tableau II}

Caractéristiques physiques et efficacités des poumons contaminés en uranium-235 utilisés pour l'étude comparative de ces poumons.

Intrinsic characteristics and efficiencies of lungs contaminated with uranium-235 used for the comparative study of these lungs.

\begin{tabular}{lccc}
\hline & ${ }^{235} \mathrm{U}$ enrichi à $93 \%$ & ${ }^{235} \mathrm{U}$ “MOX” & ${ }^{235} \mathrm{U}$ enrichi à $3 \%$ \\
\hline Activité en ${ }^{235} \mathrm{U}(\mathrm{Bq})$ & 80 & 88 & 85 \\
Masse $(\mathrm{g})$ & 1031 & 1029 & 1373 \\
Densité $\left(\mathrm{g} \mathrm{cm}^{-3}\right)$ & 0,25 & 0,25 & 0,34 \\
Efficacité absolue mesurée $\left(\times 10^{-4}\right)$ & 20,45 & 18,32 & 21,04 \\
Efficacité absolue théorique calculée $\left(\times 10^{-4}\right)$ & & 18,53 & \\
\hline
\end{tabular}

concerne la densité, des écarts de près de $40 \%$ sont constatés entre les poumons contenant $d u{ }^{235} \mathrm{U}$ enrichi à $3 \%$ et les autres jeux de poumons ; il est intéressant de noter que ces différences peuvent être corrélées avec le fournisseur de ces poumons, les poumons «MOX» et uranium enrichi à $93 \%$ n'ayant pas été fabriqués par la même société que les poumons uranium enrichi à $3 \%$. Par ailleurs, un écart de $15 \%$ entre les poumons contenant uniquement du ${ }^{235} \mathrm{U}$ enrichi à $3 \%$ et les poumons «MOX » a également pu être constaté sur l'efficacité du principal pic photoélectrique à $185,72 \mathrm{keV}$.

En parallèle de ces mesures, l'efficacité absolue théorique de notre installation a été déterminée par simulation pour une géométrie identique et des poumons réalistes dont la composition est définie par l'ICRU (1989). Cette efficacité, de $18,53 \times 10^{-4}$, est semblable à celle des poumons « MOX » mais des écarts de 10 à $15 \%$ sont observés entre cette efficacité théorique et les autres poumons uranium mesurés. Ceci laisserait donc supposer que les poumons « MOX » sont «valides » et que nos poumons contenant uniquement de l'uranium enrichi possèdent soit une activité plus importante que celle mentionnée dans le fichier certificat, soit une distribution non uniforme de l'activité.

Une intercomparaison s'avèrera donc intéressante à réaliser non seulement pour valider ces résultats mais également pour observer les différences d'étalonnage engendrées notamment par l'utilisation de poumons différents.

\section{Applications à des cas concrets de mesure in vivo}

La méthode étant validée, il est alors possible de l'utiliser pour des applications concrètes. La souplesse d'CEDIPE associée à la puissance du calcul va permettre la réalisation d'études impossibles à effectuer avec des mesures physiques 
a)

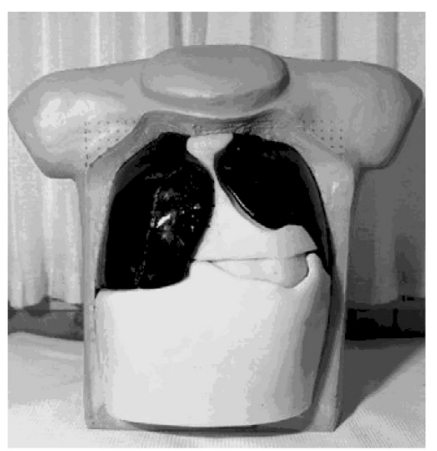

b)

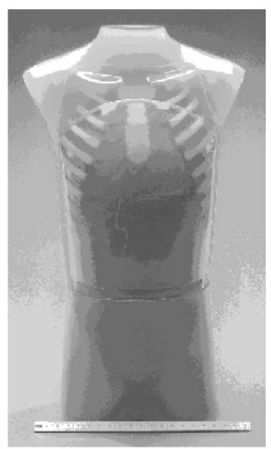

c)

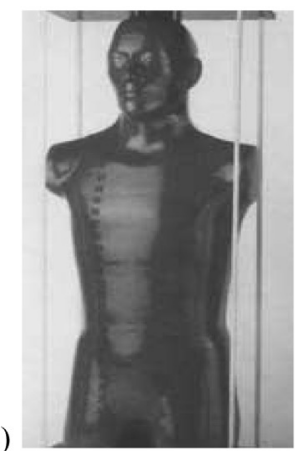

Figure 4-Fantômes physiques utilisés pour l'étalonnage de systèmes anthroporadiamétriques pulmonaires : (a) Livermore, (b) Jaeri, (c) Rando.

Physical phantoms used for the calibration of in vivo lung measurement systems: (a) Livermore, (b) Jaeri, (c) Rando.

classiques. Deux exemples des potentialités du calcul numérique sont présentés ciaprès : une étude des différents fantômes physiques utilisés pour l'étalonnage des systèmes et l'influence sur la mesure de contaminations non homogènes.

\subsection{Comparaison de différents fantômes d'étalonnage avec le fantôme de Zubal}

Afin d'étudier le potentiel d'CEDIPE dans le cadre d'étalonnages de systèmes de mesure pour les actinides, nous nous sommes placés lors des simulations en situation de mesure anthroporadiamétrique réelle sur une installation composée de quatre détecteurs ; c'est pourquoi la procédure de mesure de routine a été suivie. Par ailleurs, la personne sur laquelle la mesure virtuelle a été réalisée, a subi un examen scanner afin de permettre la reconstruction d'un fantôme numérique : il s'agit du fantôme de Zubal (Zubal et al., 1994). Le but de cette étude est de montrer l'écart que l'on pourrait obtenir sur l'estimation de l'activité pour la mesure de la personne correspondant au fantôme de Zubal en fonction du fantôme physique utilisé pour l'étalonnage du système.

\subsubsection{Matériel et méthode}

\section{Fantômes physiques}

Trois fantômes physiques d'étalonnage ont été utilisés (Fig. 4) :

- le fantôme de Livermore, décrit précédemment et déclaré par consensus fantôme standard d'étalonnage pour les hommes de type caucasien, 


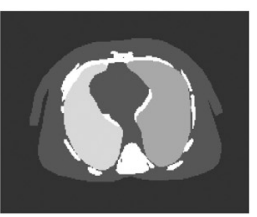

a)

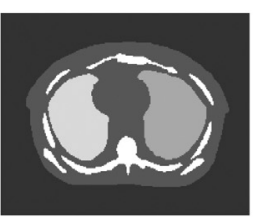

b)

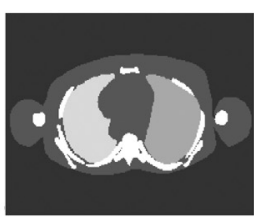

c)

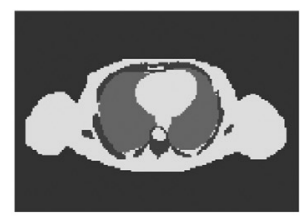

d)

Figure 5-Représentation par l'interface CEDIPE d'une coupe transverse de différents fantômes voxélisés : (a) Livermore (muni de sa plaque $n^{\circ}$ 3), (b) Jaeri (muni de sa plaque), (c) Rando, (d) Zubal.

Representation with CEDIPE software of a transverse slice of different voxel phantoms: (a) Livermore (with its overlay plate no. 3), (b) Jaeri (with its overlay plate), (c) Rando, (d) Zubal.

- le fantôme JAERI, correspondant à l'homme standard japonais (Shirotani, 1988),

- le fantôme RANDO (Beau et Le Grand, 1970), premier fantôme à avoir été utilisé pour l'étalonnage des systèmes d'anthroporadiamétrie basse énergie et qui n'est plus utilisé à l'heure actuelle.

Réalisation des fantômes numériques

Pour la reconstruction des fantômes numériques voxélisés (Fig. 5), la procédure décrite précédemment a été utilisée: (i) acquisition des images scanner des fantômes physiques, (ii) segmentation de ces images. Les tailles des pixels de ces fantômes sont respectivement de $0,18 \times 0,18 \mathrm{~cm}^{2}$ pour le Livermore, $0,17 \times$ $0,17 \mathrm{~cm}^{2}$ pour le Jaeri et $0,20 \times 0,20 \mathrm{~cm}^{2}$ pour le Rando ; la taille en $Z$ de chaque voxel est de $1 \mathrm{~cm}$, ce qui correspond à l'épaisseur de coupe.

Le fantôme de Zubal est quant à lui un fantôme directement voxélisé ; reconnu internationalement, il a été obtenu par l'équipe de G. Zubal (http://noodle.med.yale.edu/phant.html) à partir de coupes tomodensitométriques d'un homme de $178 \mathrm{~cm}$ pour $70 \mathrm{~kg}$ (Zubal et al., 1994), donc assez proche de l'homme standard (ICRP, 2002). La taille des voxels de ce fantôme est de 0,36 × $0,36 \times 0,36 \mathrm{~cm}^{3}$.

Chaque voxel des fantômes numériques est constitué d'un matériau équivalent tissu (muscle, graisse, os, poumons, ou matériau $50 \%$ muscle - $50 \%$ graisse pour les plaques extrathoraciques), défini par l'ICRU (1989). Une activité simulée de 
$70 \mathrm{kBq}$ en ${ }^{241} \mathrm{Am}$ ou de $80 \mathrm{~Bq}$ en ${ }^{235} \mathrm{U}$ est uniformément répartie dans le volume pulmonaire de chacun des fantômes.

Le système de mesure simulé est composé de 4 détecteurs germanium haute pureté, avec la même configuration que celle proposée par Kramer (Kramer et al., 1998) (cf. Fig. 2). Chaque mesure est simulée avec un temps de comptage de 1000 secondes.

\section{Calcul de l'épaisseur thoracique des fantômes}

L'épaisseur de la paroi thoracique du fantôme de Zubal est calculée à partir de l'équation (1) déterminée par Vickers pour un homme de poids $P$ et de taille $T$, avec une contamination en ${ }^{241} \mathrm{Am}$ (énergie considérée : 59,5 keV) et 3 détecteurs germanium, configuration la plus proche de celle utilisée pour la simulation réalisée ici (Vickers, 1996).

$$
C W T 50 / 50(\mathrm{~cm})=\left[4,81 \times \frac{P(\mathrm{~kg})}{T(\mathrm{~cm})}+0,85\right] \pm 0,42 .
$$

Cette équation rapporte l'épaisseur thoracique moyenne de la personne à mesurer à une épaisseur équivalente en atténuation à un matériau $50 \%$ muscle - $50 \%$ graisse, afin de pouvoir directement évaluer la plaque extrathoracique à ajouter au fantôme physique la plus appropriée pour l'étalonnage. Ainsi, d'après cette équation, l'homme de $178 \mathrm{~cm}$ pour $70 \mathrm{~kg}$ ayant servi à la modélisation du fantôme de Zubal présente une épaisseur thoracique moyenne de 2,74 $\pm 0,42 \mathrm{~cm}$. Afin de couvrir toute cette gamme d'épaisseurs, différentes configurations des fantômes d'étalonnage représentant des morphologies équivalentes ont été choisies; ainsi les fantômes Livermore et Jaeri ont été équipés de différentes plaques extrathoraciques supplémentaires, d'un matériau équivalent à $50 \%$ muscle $-50 \%$ graisse.

\subsubsection{Comparaison des fantômes numériques voxélisés}

Les caractéristiques morphologiques des différents fantômes sont présentées au tableau III. Les tailles et poids des différents fantômes physiques sont extraits du rapport 48 de l'ICRU (1992). Leurs volumes pulmonaires ont été calculés à l'aide du logiciel DOSIGRAY ${ }^{\circledR}$, ainsi que les différentes distances présentées. Les épaisseurs de paroi thoracique définies ici et réalisées au milieu des poumons correspondent à des épaisseurs géométriques moyennes, c'est-à-dire directement mesurées sur les images scanner. Il est intéressant de noter que les épaisseurs de paroi thoracique du Livermore et du Jaeri sont indépendantes de la taille et du poids de chaque fantôme ; ces paramètres, donnés à titre indicatif, correspondent en fait à ceux des personnes ayant servi à l'élaboration des fantômes physiques et ne sont donc pas représentatifs de la morphologie considérée. 
Tableau III

Caractéristiques morphologiques des différents fantômes et comparaison avec l'homme de référence (ICRP, 2002).

Morphological characteristics of different phantoms and comparison with the Reference Man (ICRP, 2002).

\begin{tabular}{|c|c|c|c|c|c|c|c|c|}
\hline & \multicolumn{3}{|c|}{ Livermore } & \multicolumn{2}{|l|}{ Jaeri } & \multirow[t]{2}{*}{ Rando } & \multirow[t]{2}{*}{ Zubal } & \multirow{2}{*}{$\begin{array}{c}\text { Reference } \\
\text { Man CIPR } 89\end{array}$} \\
\hline & Plaque 1 & Plaque 2 & Plaque 3 & Sans plaque & Plaque & & & \\
\hline Taille corps entier $(\mathrm{cm})$ & & 177 & & 168 & & 175 & 178 & 176 \\
\hline Poids corps entier $(\mathrm{kg})$ & & 76 & & 63,5 & & 73,5 & 70 & 73 \\
\hline $\begin{array}{l}\text { Épaisseur thoracique } \\
\text { géométrique }(\mathrm{cm})\end{array}$ & 2,4 & 3,3 & 3,6 & 2,0 & 3,8 & 3,8 & 2,7 & 3 \\
\hline Longueur des poumons $(\mathrm{cm})$ & & 22 & & 28 & & 25 & 20 & 28,5 \\
\hline $\begin{array}{l}\text { Épaisseur antéro-postérieure } \\
\text { des poumons }(\mathrm{cm})\end{array}$ & & 22 & & 15 & & 16 & 17,5 & 20 \\
\hline Volume pulmonaire $\left(\mathrm{cm}^{3}\right)$ & & 4075 & & 3691 & & 3436 & 2910 & 4172 \\
\hline
\end{tabular}

Il convient de noter que l'épaisseur thoracique mesurée du fantôme de Zubal et son équivalent calculé par l'équation de Vickers sont identiques ; ce résultat est surprenant du fait que les deux grandeurs ne sont pas les mêmes, la première étant une valeur géométrique (la résolution du fantôme de Zubal étant de $0,36 \mathrm{~cm}$ ) et la seconde étant une grandeur équivalente en matériau $50 \%$ muscle - $50 \%$ graisse, ce qui prouve les limites des équations bioparamétriques en général.

Il est constaté qu'aucun de ces différents fantômes physiques ne correspond exactement à l'homme de référence défini dans la publication 89 de la CIPR (ICRP, 2002), le fantôme Livermore muni de la plaque 2 paraissant être le fantôme le plus proche de la morphologie de référence.

\subsubsection{Résultats et discussion}

Les résultats de la réponse des différents fantômes d'étalonnage, pour des énergies comprises entre 26 et $185 \mathrm{keV}$ et normalisés par rapport au fantôme de Zubal, sont donnés à la figure 6 . Les réponses des quatre détecteurs ont été sommées pour chaque fantôme ; les incertitudes sont données à un écart-type $(1 \sigma)$.

Pour toutes les configurations sauf pour le fantôme Livermore muni de la plaque 1 et le fantôme Jaeri sans plaque et quelle que soit l'énergie, une sous-évaluation systématique de l'efficacité de détection par rapport au fantôme de Zubal est observée (environ $30 \%$ ). Ceci aurait pour conséquence, en cas de mesure de la personne ayant servi à la modélisation du fantôme de Zubal, de surévaluer l'activité présente en ${ }^{241} \mathrm{Am}$ ou en ${ }^{235} \mathrm{U}$ d'environ $30 \%$. Ces résultats peuvent être principalement expliqués par l'épaisseur extrathoracique plus petite du fantôme de 


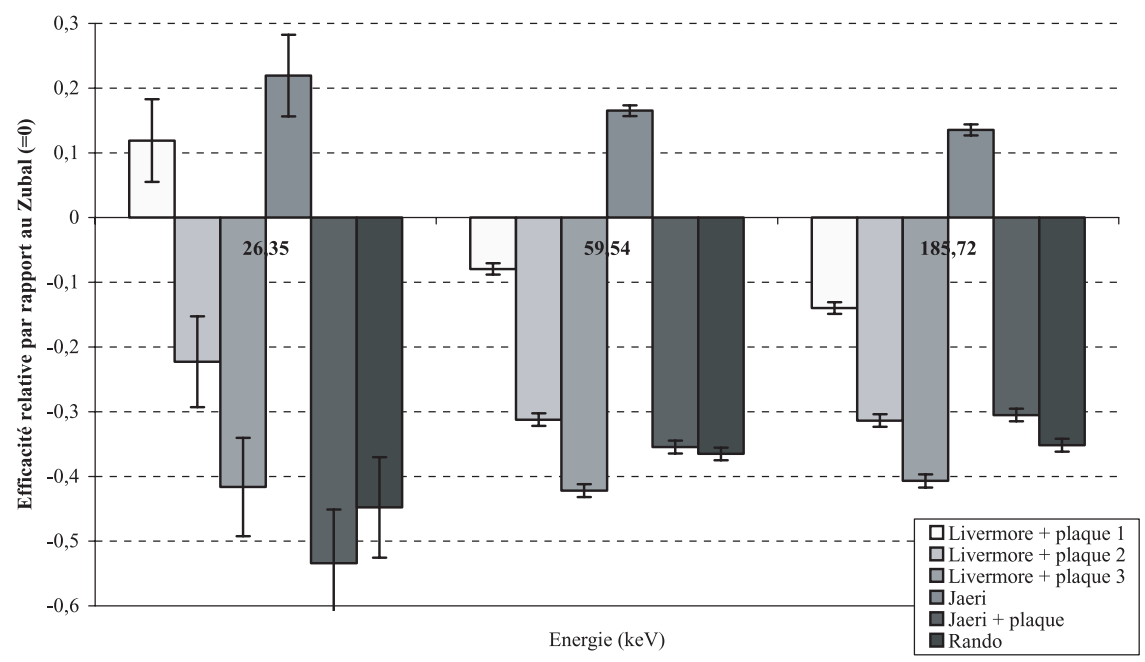

Figure 6 - Efficacités comparées entre les différents fantômes d'étalonnage normalisés par rapport au fantôme de Zubal.

Comparison of the efficiencies for the different calibration phantoms normalised to the Zubal phantom.

Zubal par rapport aux fantômes d'étalonnage utilisés : en effet, l'épaisseur de la paroi thoracique du fantôme de Zubal est de l'ordre de 2,7 cm alors qu'elle varie entre 3,3 et 3,8 cm pour les autres fantômes, induisant à basse énergie une diminution des photons absorbés dans les tissus pour le fantôme de Zubal.

Par ailleurs, l'histogramme montre une surestimation de la réponse du Jaeri sans plaque par rapport à celle du fantôme de Zubal quelle que soit l'énergie ; quant au Livermore équipé de la plaque 1, il surestime la réponse du fantôme de Zubal à $26 \mathrm{keV}$ puis la sous-estime pour des énergies supérieures. Ces résultats peuvent être expliqués non seulement par une épaisseur de paroi thoracique plus fine pour ces deux fantômes ( $2 \mathrm{~cm}$ pour le Jaeri et $2,4 \mathrm{~cm}$ pour le Livermore muni de la plaque 1), mais aussi par des formes différentes de poumons, tant en longueur qu'en profondeur.

$\mathrm{Au}$ vu de ces résultats et considérant l'équipement de nombreux laboratoires, le fantôme Livermore muni de la plaque 1 semble donc le plus approprié pour l'étalonnage du système si la personne correspondant au Zubal était mesurée. Si les protocoles suivis dans les laboratoires d'Analyse et de biologie médicale (LABM) avaient été utilisés (Bérard et Aussel, 1994), compte tenu du poids et de la taille de la personne représentée par le fantôme de Zubal, la plaque 2 du Livermore aurait été privilégiée pour l'étalonnage du système, induisant ainsi une plus importante surévaluation de l'activité présente dans les poumons. 
Cette étude montre ainsi l'intérêt de réaliser des étalonnages numériques des installations de comptage réelles avec des fantômes voxélisés de morphologie proche de celle des personnes à mesurer.

\subsection{Approches de contaminations inhomogènes}

Une des hypothèses de l'étalonnage des systèmes de mesure basse énergie repose sur le postulat d'une répartition homogène de la contamination dans les poumons, dû en partie à des raisons techniques de fabrication des poumons sources mais également au fait qu'il existe peu de données concernant la distribution spatiale des particules au sein des poumons.

Afin de mieux appréhender l'influence d'une hétérogénéité de contamination sur les facteurs d'étalonnage, toujours estimés pour une contamination homogène, nous avons donc réalisé par simulation des contaminations pulmonaires non homogènes en considérant un modèle de référence. Le but de cette étude n'était pas de modéliser exactement une contamination réaliste mais seulement d'en réaliser une approche.

La CIPR a montré que les poussières après inhalation se déposaient préférentiellement dans la région alvéolaire (ICRP, 1994). Afin de « simuler » une telle contamination, une contamination en couches a été considérée.

\subsubsection{Méthode}

Les alvéoles étant préférentiellement situées à la périphérie des lobes pulmonaires, un modèle a été réalisé en contaminant la périphérie des poumons. Pour cela, des marges de $2 \mathrm{~cm}, 1 \mathrm{~cm}, 7 \mathrm{~mm}$ et $5 \mathrm{~mm}$ à l'intérieur des poumons du fantôme Livermore ont été créées à l'aide du logiciel DOSIGRAY ${ }^{\circledR}$ (Fig. 7).

L'activité ( $70 \mathrm{kBq}$ en ${ }^{241} \mathrm{Am}$ et $80 \mathrm{~Bq}$ en ${ }^{235} \mathrm{U}$ ) a ensuite été répartie de façon homogène dans ces marges à l'aide d'CEDIPE, le reste des poumons étant dépourvu de contamination. Afin d'étudier l'influence de cette répartition inhomogène, un système de comptage virtuel composé d'un seul détecteur germanium a été positionné au-dessus du milieu du poumon droit et les spectres calculés ont été comparés à ceux calculés dans la géométrie de référence, c'est-àdire avec une contamination homogène dans tout le poumon. Le temps de comptage simulé était de 1000 secondes.

\subsubsection{Résultats et discussion}

Les résultats sont reportés sur la figure 8 (incertitudes données à $1 \sigma$ ). La forme de l'histogramme montre une nette diminution du rapport entre contaminations surfacique et homogène, avec l'énergie : de l'ordre de $30 \%$ d'écart à $15,5 \mathrm{keV}$, 
a)

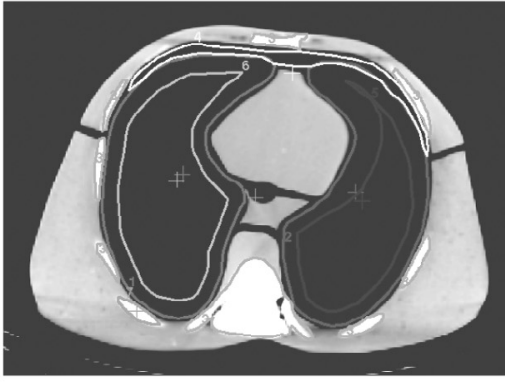

b)

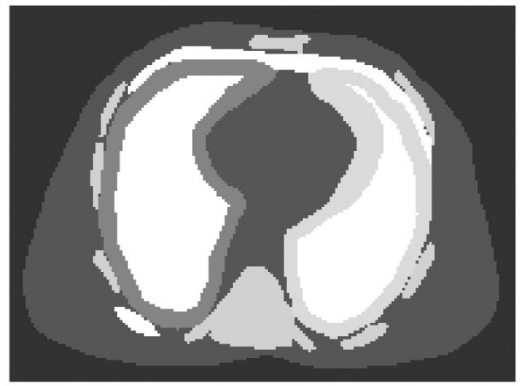

Figure 7 - Visualisation par DOSIGRAY ${ }^{\circledR}$ (a) et $\operatorname{CDIPE}(\mathrm{b})$ du fantôme Livermore avec une marge pulmonaire de $1 \mathrm{~cm}$.

Vizualisation with DOSIGRAY ${ }^{\circledR}$ (a) and $\operatorname{EDIPE}$ (b) of Livermore phantom with $1 \mathrm{~cm}$ lung margin.

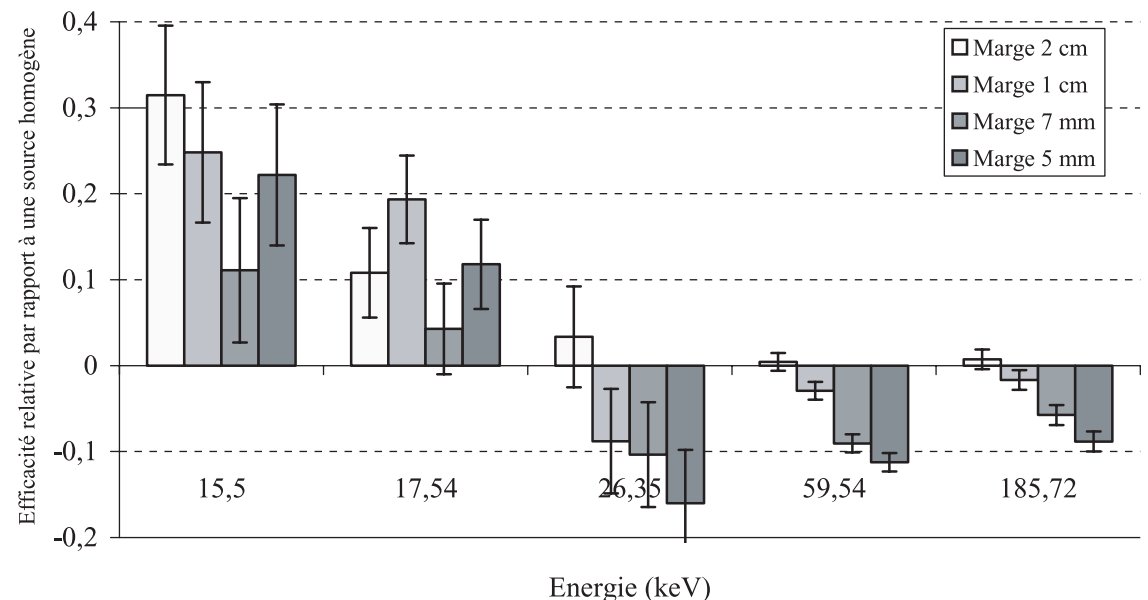

Figure 8 - Efficacités comparées entre les contaminations en couches et une contamination homogène. Comparison of the efficiencies for shell lung contaminations normalised to uniform lung contamination.

le rapport chute à moins de $15 \%$ à $26,35 \mathrm{keV}$, même s'il est difficile de tirer des conclusions du fait des incertitudes importantes (données à $1 \sigma$ ). Pour des énergies supérieures à $26,35 \mathrm{keV}$, il est constaté qu'une contamination surfacique de $2 \mathrm{~cm}$ est plus proche d'une contamination homogène qu'une contamination surfacique de $5 \mathrm{~mm}$; ce résultat est a priori logique du fait des volumes différents dans lesquels sont réparties les sources ( $c f$. Tab. IV). 


\section{Tableau IV}

Volumes des sources pour les différentes configurations estimés à l'aide de DOSIGRAY ${ }^{\circledR}$, et activité volumique normalisée par rapport à une contamination homogène.

Volume of the sources for different configurations, estimated by DOSIGRAY ${ }^{\circledR}$, and volumic activity normalised to uniform lung contamination.

\begin{tabular}{lccccc}
\hline & Marge 0,5 cm & Marge 0,7 cm & Marge 1 cm & Marge 2cm & Homogène \\
\hline Volume source poumon droit $\left(\mathrm{cm}^{3}\right)$ & 487 & 852 & 1139 & 1742 & 2294 \\
Volume source poumon gauche $\left(\mathrm{cm}^{3}\right)$ & 405 & 739 & 946 & 1442 & 1781 \\
$\begin{array}{l}\text { Activité volumique normalisée par } \\
\text { une contamination homogène }\end{array}$ & 4,57 & 2,56 & 1,95 & 1,28 & 1,00 \\
\hline
\end{tabular}

Toutefois peu de différences sont observées entre une contamination surfacique et une contamination homogène, les écarts étant quasiment toujours inférieurs à $5 \%$ pour les marges de 1 et $2 \mathrm{~cm}$ et à $10 \%$ pour les marges plus fines, ce qui a priori est assez étonnant. Ceci peut s'expliquer par une sorte de compensation des activités : en effet, la même activité est placée soit dans la totalité du poumon soit dans les marges, ce qui implique dans ce dernier cas une concentration volumique de l'activité plus élevée. En effet, une activité volumique près de 5 fois plus élevée qu'avec une contamination homogène a été répartie dans la marge de $5 \mathrm{~mm}$, du fait du volume réduit de cette dernière ( $c f$. Tab. IV). L'activité étant concentrée dans les marges et la contamination positionnée dans la partie antérieure des poumons se trouvant très proche du détecteur, plus de photons parviendront au détecteur que dans le cas d'une contamination homogène, en particulier à basse énergie parce que les photons émis en profondeur dans les poumons seront absorbés pendant la traversée du volume pulmonaire. Plus l'énergie des photons deviendra importante, moins les photons seront absorbés dans les tissus, engendrant ainsi une diminution des écarts. Ce phénomène s'atténue lorsque l'énergie des photons augmente, du fait de la diminution de l'absorption et de l'angle solide (Razafindralambo, 1995).

En conclusion, cette première approche semblerait montrer que pour des énergies supérieures à $20 \mathrm{keV}$, il existerait peu de variations entre des contaminations inhomogènes et homogène.

\section{Conclusion}

L'interface CEDIPE a été développée afin de rendre possible l'utilisation de fantômes numériques voxélisés pour l'étalonnage de systèmes de mesures anthroporadiamétriques. Dans un premier temps, la validation de l'outil effectuée avec le fantôme de Livermore a été démontrée, les écarts entre simulation et mesure étant inférieurs à $20 \%$; cette étape a également permis de montrer le potentiel de 
la technique pour l'étude des erreurs systématiques, notamment la sensibilité de positionnement du détecteur. Dans un second temps, l'application de cet outil à la comparaison de fantômes servant à l'étalonnage des systèmes de mesure pulmonaire a montré le potentiel de cette technique pour l'amélioration des étalonnages et la meilleure maîtrise des incertitudes liées à la mesure ; cette étude a notamment montré les limites de l'utilisation des équations bioparamétriques et donc prouvé l'intérêt de réaliser des étalonnages numériques des installations de comptage. Enfin, des approches de contaminations inhomogènes ont permis d'entrevoir les possibilités offertes par la simulation pour une meilleure prise en compte de paramètres jusqu'alors impossibles à étudier avec des fantômes physiques, concernant notamment la distribution spatiale de la contamination pulmonaire ; il semblerait notamment que, pour des énergies supérieures à $26 \mathrm{keV}$, il n'y ait pas de différence significative entre une contamination homogène et une contamination périphérique, les modèles étudiés étant toutefois très simplistes. Il conviendra bien entendu de confirmer ou non ces résultats avec des contaminations plus réalistes. Le potentiel de l'outil EDDIPE sera également exploité dans des études complémentaires, notamment la modélisation d'installations réelles en vue de l'étalonnage numérique de chaînes anthroporadiamétriques afin de réduire les incertitudes liées à l'utilisation de fantômes physiques d'étalonnage.

Remerciements. Ce travail est réalisé dans le cadre d'un programme d'intérêt commun IRSN-COGEMA.

\section{RÉFÉRENCES}

Aubineau-Lanièce I., de Carlan L., Clairand I., Lemosquet A., Chiavassa S., Pierrat N., Franck D. (2004) Current developments at IRSN on computational tools dedicated to dose assessment for both internal and external exposure. Papier invité, conférence ICRS-RPS 2004, 9-14 mai 2004, Madeira, accepté.

Beau P.G., Le Grand J. (1970) Utilisation d'un fantôme équivalent-tissus pour l'estimation de la contamination radioactive et de la dose absorbée au niveau de certains organes, Radioprotection 5, 167-174.

Bérard P., Aussel J.P. (1994) Surveillance anthroporadiamétrique X et gamma des travailleurs exposés aux composés de l'uranium à l'aide de détecteurs coaxiaux, IN Proc. Journées de spectrométrie gamma et X 93, St Rémy les Chevreuses, France, 1993. DTA/DAMRI ed. Rapport CEA-N2756, 259-263.

Borisov N., Franck D., de Carlan L., Laval L. (2002) A new graphical user interface for fast construction of computation phantoms and MCNP calculations: application to calibration of in vivo measurement systems, Health Phys. 83, 272-279.

Breismeister J.F. (2000) MCNP ${ }^{\mathrm{TM}}$. A general Monte Carlo N-particle transport code, version 4c. LANL Memorandum. Los Alamos National Laboratory.

de Carlan L., Aubineau-Lanièce I., Lemosquet A., Borissov N., Jourdain J.R., Jeanbourquin D., Le Guen B., Franck D. (2003) Application of new imaging and calculation techniques for the activity and dose assessment in the case of a ${ }^{106} \mathrm{Ru}$ contaminated wound, Rad. Prot. Dosim. 105, 219-224. 
Franck D., de Carlan L., Bérard P., Dousse C., Pihet P., Razafindralambo N., Soulié R. (1997) Les mesures anthroporadiamétriques dans les basses énergies : évolution technologique et bases des recherches futures, Radioprotection 32, 685-696.

Franck D., Laval L., Borissov N., Guillierme P., Bordy J.M. (2001) Development of voxelised numerical phantoms using MCNP Monte Carlo code: application to in vivo measurement, Radioprotection 36, 77-86.

Franck D., Borissov N., de Carlan L., Pierrat N., Genicot J. L., Etherington G (2003) Application of Monte Carlo calculations to calibration of anthropomorphic phantoms used for activity assessment of actinides in lungs, Rad. Prot. Dosim. 105, 403-408.

Griffith R.V., Dean P.N., Anderson A.L., Fisher J.C. (1978) A tissue-equivalent torso phantom. Proceedings of an IAEA Conference, Advances in radiation monitoring. Vienna, IAEA ; IAEASM-229/56, 4493-4504.

Hunt J.G., Dantas B.M., Lucena E. (1998) Calibration of an in vivo measurement system using a voxel phantom, Rad. Prot. Dosim. 79, 425-427.

ICRP Publication 66 (1994) Human respiratory tract model for radiological protection, Ann. ICRP 23 (2).

ICRP Publication 89 (2002) Basic anatomical and physiological data for use in radiological protection: Reference values, Ann. ICRP 32 (3-4).

ICRU (1989) Tissue substitutes in Radiation Dosimetry and Measurements. ICRU Report 44, ICRU Publication, Bethesda.

ICRU (1992) Phantoms and computational models in therapy, diagnosis and protection. ICRU Report 48, ICRU Publication, Bethesda.

Kramer G.H., Hauck B.M. (2000) Intercomparison of JAERI torso lung sets, Rad. Prot. Dosim. 88 311-317.

Kramer G.H., Hauck B.M., Allen S.A. (1998) Comparison of the LLNL and JAERI torso phantoms using Ge detectors and phoswich detectors, Health Phys. 74, 594-601.

Mallett M.W., Hickman D.P., Krutchen D.A., Poston J.W. (1995) Development of a method for calibrating in vivo measurement systems using magnetic resonance imaging and Monte Carlo computation, Health Phys. 68, 773-785.

Newton D., Wells A.C., Mizushita S., Toohey R.E., Sha J.Y., Jones R., Jefferies S.J., Palmer H.E., Rieksts G.A., Anderson A.L., Campbell G.W. (1985) The Livermore phantom as a calibration standard in the assessment of plutonium in lungs. Proceedings of a Symposium, Assessment of Radioactive Contamination in Man, Paris, 19-23 november 1984, organized by IAEA in cooperation with WHO.

Razafindralambo N. (1995) Limite de détection de la spectrométrie X pour l'anthroporadiamétrie pulmonaire du plutonium: Analyse et perspectives de développement. Thèse de l'Université Paul Sabatier de Toulouse.

Shirotani T. (1988) Realistic torso phantom for calibration of in vivo transuranic-nuclide counting facilities, J. Nucl. Sci. Technol. 25, 875-883.

Spitz H., Glover S., Liu N., Smith B., Hickman D., Kruchten D., Anderson L. (1994) Measurement of the attenuation coefficient for Livermore thoracic phantom lungs fabricated using contemporary materials, Health Phys. 67, 39-46.

Vickers L.R. (1996) The gender-specific chest wall thickness prediction equations for routine measurements of ${ }^{239} \mathrm{Pu}$ and ${ }^{241} \mathrm{Am}$ within the lungs using HPGe detectors, Health Phys. 70, 346-357.

Zubal I.G., Harrell C.R., Smith E.O., Rattner Z., Gindi G., Hoffer P.B. (1994) Computerized threedimensional segmented human anatomy, Med. Phys. 21, 299-302. 